\title{
Threshold behavior of positronium formation in positron-alkali scattering
}

\author{
A. V. Lugovskoy, R. Utamuratov, A. S. Kadyrov, A. T. Stelbovics, and I. Bray \\ ARC Centre for Antimatter-Matter Studies, Curtin University, GPO Box U1987, Perth, WA 6845, Australia
}

(Dated: April 12, 2013)

\begin{abstract}
We consider positron scattering on the alkali atoms of $\mathrm{Li}, \mathrm{Na}$, and $\mathrm{K}$ at very low energies, where only the elastic scattering and positronium formation in the ground state are the two open channels. Utilising the recently developed two-center convergent close coupling method [Lugovskoy et. al., Phys. Rev. A 82, 062708 (2010)] we investigate the behavior of the cross sections as the impact energy goes to zero and demonstrate their convergence. The study sets quantitative benchmarks for any rigorous theoretical treatment of the collision problems.
\end{abstract}

PACS numbers: $34.80 .-\mathrm{i}$

\section{INTRODUCTION}

In 1948 Wigner showed that the cross-section energy dependence near the threshold energy does not depend on the particular reaction mechanism, but is governed only by the long-range interaction of the reacting particles [1]. For the case of a final state with two fragments Wigner gave a solution for three typical cases of long-range potentials. The derived threshold laws were found to be applicable for the description of a rich variety of collision processes [2]. Initially, studies of threshold effects utilized collisions involving electrons [3] and positrons [4-7]. Their longer wavelengths allowed characterization of the scattering process using few parameters over a substantial energy range. More recently experimental techniques have evolved for direct study of threshold effects in ultracold collisions with heavier particles such as atomic nuclei [8], fermionic atoms [9] and molecular fragments [10].

In the case of positron-alkali scattering the positronium (Ps) formation threshold lies at zero energy. For such exothermic reactions the Wigner law predicts that the $S$ wave cross sections behave as $1 / k_{0}$, where $k_{0}$ is the wave number of the incident positron, and hence be infinite at threshold. For elastic scattering the cross section should converge to a constant. Watts and Humberston [11] and Humberston and Watts [12] conducted Kohn variational calculations for positron-lithium collisions and observed the rise in the $s$-wave cross section for positronium formation with decreasing momentum of the incident positron. They interpreted this observation in terms of the Wigner threshold law.

Similar exothermic reaction systems occur elsewhere. Using the Kohn variational method Armour and Chamberlain [13] have found such results for the elastic and rearrangement cross sections in hydrogen-antihydrogen scattering. A similar $1 / k_{0}$-dependence of the cross section for $\mathrm{H}$ formation in Ps collisions with protons was reported by Mitroy [14]. A simple explanation of such behavior was given by McAlinden et al. [5] in their paper on positron-lithium scattering. But the argument is of general character. They point out that the observed regularities are due to the dominating kinematic $1 / k_{0}$ factor in the expression for the cross section in the absence of amplitude dependence on $k_{0}$. However, this needs to be established in a fully convergent formalism.

There are several theoretical studies of positron scattering from alkali targets at energies above $1 \mathrm{eV}$ [15-21], where they could be tested against experiment [22-24]. Threshold behavior in positron scattering from lithium was studied in Refs. [5, 11, 12, 25]. It was shown that both elastic and $\mathrm{Ps}_{\mathrm{S}}(1 \mathrm{~s})$ formation cross sections obey Wigner threshold laws. In Kohn variational calculations up to two hundred and twenty of short-range correlation terms were used for the trial wave functions [12]. However, the absolute values of the Ps formation cross sections differ by orders of magnitude depending on the chosen model parameters $[11,12]$. In the case of closecoupling calculations up to fourteen states were used and no convergence was established for these quantities which also varied in wide limits [5, 25]. Recently, Ward and Shertzer [20] and Ward et al. [21] applied the hyperspherical hidden crossing method to Ps formation in positronlithium scattering and found a rise in the s-wave cross section for Ps formation as the momentum of the incident positron is decreased. A similar result was also obtained for positron-sodium scattering [26].

In this work we present the results of the convergent close-coupling (CCC) calculations for positron scattering from the alkali atoms of $\mathrm{Li}, \mathrm{Na}$ and $\mathrm{K}$, at very low projectile energies. The first two atoms are able to weakly bind a positron while the third atom, K, is not [27]. The existence of these states was shown theoretically with the use of variational calculations [28]. Our goal is to present convergent Ps-formation and elastic cross sections at the very low incident positron energies, as well as confirming Wigner's threshold law for Ps(1s) formation utilizing a fully convergent formalism.

\section{THEORY}

The details of the two-center CCC formalism for positron scattering have been given in Refs. [29, 30]. The lighter alkali atoms are readily treated by the frozen-core Hartree-Fock model of one valence electron above a relatively inert core [31]. Core polarizability is treated using phenomenological polarization potentials. To incorporate explicit Ps-formation, it is convenient to replace the 
non-local exchange part of the core potential by a local approximation [30, 32]. Another approximations is to neglect electron exchange between the Ps electron and the target core electrons. Given the relatively inert closed shell core we expect the effect of these approximations to be small.

The one-electron target $(\mathrm{T})$ Hamiltonian $H_{\mathrm{T}}$ is diagonalised for each orbital angular momentum $l \leq l_{\max }$ to obtain target pseudostates using

$$
\left\langle\phi_{f}^{(\mathrm{T})}\left|H_{\mathrm{T}}\right| \phi_{i}^{(\mathrm{T})}\right\rangle=\epsilon_{f}^{(\mathrm{T})} \delta_{f i},
$$

where the $\phi_{n}^{(\mathrm{T})}(r)$ are linear combinations of the complete Laguerre basis functions

$$
\begin{aligned}
\xi_{n, l}^{(\lambda)}(r)= & \left(\frac{\lambda(n-1) !}{(2 l+1+n) !}\right)^{1 / 2} \\
& \times(\lambda r)^{l+1} \exp [-\lambda r / 2] L_{n-1}^{2 l+2}(-\lambda r),
\end{aligned}
$$

and where $L_{n-1}^{2 l+2}(x)$ is the associated Laguerre polynomial, and $n$ ranges from 1 to the basis size $N$. With increasing $N$ the negative-energy states converge to the true discrete eigenstates, while the positive-energy states yield an increasingly dense discretization of the target continuum. Explicit inclusion of Ps (pseudo)states also requires diagonalization of the Ps Hamiltonian in a Laguerre basis

$$
\left\langle\phi_{f}^{(\mathrm{Ps})}\left|H_{\mathrm{Ps}}\right| \phi_{i}^{(\mathrm{Ps})}\right\rangle=\epsilon_{f}^{(\mathrm{Ps})} \delta_{f i} .
$$

To get the scattering cross sections we solve the set of momentum-space coupled-channel equations for transition matrix elements $\left(\gamma^{\prime}=1, \ldots, N^{(\mathrm{T})}+N^{(\mathrm{Ps})}\right)$

$$
\begin{aligned}
T_{\gamma^{\prime}, \gamma}\left(\boldsymbol{q}_{\gamma^{\prime}}, \boldsymbol{q}_{\gamma}\right)= & V_{\gamma^{\prime}, \gamma}\left(\boldsymbol{q}_{\gamma^{\prime}}, \boldsymbol{q}_{\gamma}\right)+\sum_{\gamma^{\prime \prime}}^{N^{(\mathrm{T})}+N^{(\mathrm{Ps})}} \int d \boldsymbol{q}_{\gamma^{\prime \prime}} \\
& \times \frac{V_{\gamma^{\prime}, \gamma^{\prime \prime}}\left(\boldsymbol{q}_{\gamma^{\prime}}, \boldsymbol{q}_{\gamma^{\prime \prime}}\right) T_{\gamma^{\prime \prime}, \gamma}\left(\boldsymbol{q}_{\gamma^{\prime \prime}}, \boldsymbol{q}_{\gamma}\right)}{E+i 0-\epsilon_{\gamma^{\prime \prime}}-q_{\gamma^{\prime \prime}}^{2} /\left(2 M_{\gamma^{\prime \prime}}\right)},
\end{aligned}
$$

where $E$ is the total energy, $\boldsymbol{q}_{\gamma}$ is the momentum of the free particle $\gamma$ relative to the c.m. of the bound pair in channel $\gamma$ (T or Ps), $\epsilon_{\gamma}$ is the corresponding pseudoenergy of the bound pair, $M_{\gamma}$ is its reduced mass and $V_{\gamma^{\prime}, \gamma}$ are the effective potentials [30].

One of the strengths of the two-center CCC formalism is the ability to check for internal consistency. Both expansions approach completeness with increasing $N$, but are not orthogonal to each other. The unitarity of the close-coupling formalism ensures that double-counting cannot occur, but the potential overcompleteness manifests itself through ill-conditioned linear equations when solving Eq. (4). Thus, we cannot arbitrarily increase our basis sizes, but need to be particularly careful in demonstrating convergence. At the very low energies considered here we only have two open states, the target ground state and $\operatorname{Ps}(1 \mathrm{~s})$. All other states are closed, and represent virtual excitation, ionization and Ps formation (in excited states). These virtual effects can be very large, and due to the completeness of the Laguerre basis, can be treated by either center. Internal consistency is established when widely varying choices of expansions on the two centers yield much the same results.

The critical aspect of the CCC approach is to demonstrate convergence at each energy of the projectile with increasing basis size parameters $N$ and $l_{\max }$, for specified exponential fall-off $\lambda$. We are free to vary $N$ and $\lambda$ for each $l$, for both the target and Ps. This creates considerable flexibility, but for the purpose of a clear presentation of the convergence we take $\lambda_{l}^{(\mathrm{Ps})}=1$ (yields exact Ps $(1 \mathrm{~s}$ ) with $\left.N^{(\mathrm{Ps})}=1\right)$, and $\lambda_{l}^{(\mathrm{T})}=4$ in all presented calculations. Furthermore, following a series of calculations we have chosen the $N_{l}^{(\mathrm{T})}=25-l$ to ensure a sufficiently large number of states to yield an accurate alkali target structure and convergence in the scattering calculations. We will demonstrate convergence by variation of $l_{\max }$ and the number of Ps states.

\section{RESULTS}

For the three alkali targets considered we perform calculations over the energy range from $10^{-5}$ to $1 \mathrm{eV}$. The lower limit was chosen to clearly demonstrate the threshold behavior of Ps-formation. The upper limit is somewhat arbitrary, but ensures that only elastic scattering and $\operatorname{Ps}(1 \mathrm{~s})$ formation remain the only open channels over the entire energy range. The emphasis on Ps-formation at the very low energies allows us to consider only the zeroth partial wave. Our calculations show that the next partial wave has a contribution that begins to be commensurate with the zeroth partial wave above $10^{-3} \mathrm{eV}$.

As a starting point we obtained convergence by taking only the $\operatorname{Ps}(1 \mathrm{~s})$ state and increasing the $l_{\max }$ of the target. Given that explicit $\operatorname{Ps}(1 \mathrm{~s})$ formation is already included, surprisingly we found that a large $l_{\max }=8$ was necessary. These calculations are labeled $\operatorname{CCC}\left(25_{8}, 1\right)$. In the case of lithium, sodium and potassium the total number of atomic states included in the close-coupling expansion is 188,186 , and 184, respectively. These calculations serve as the benchmark results for the zeroth partial wave since all of the required boundary conditions are satisfied, and the effects of virtual Ps-formation and target excitation and ionization are incorporated by the closed large- $l$ target states.

In Fig. 1 the positron-lithium results are presented. The upper panel shows the very low-energy behavior of the $\operatorname{Ps}(1 \mathrm{~s})$ cross section $\sigma_{\mathrm{Ps}}$ multiplied by the positron momentum $k_{0}$, while the lower panel presents the elastic cross section $\sigma_{\mathrm{el}}$. As expected, both $k_{0} \sigma_{\mathrm{Ps}}$ and $\sigma_{\mathrm{el}}$ tend to constant values as the positron energy goes to zero. To demonstrate the remarkable nature of the convergence with increasing $l$ of the lithium states, we also present the $\operatorname{CCC}\left(25_{4}, 1\right)$ calculations, which are roughly an order of magnitude above the converged results. This is rather 

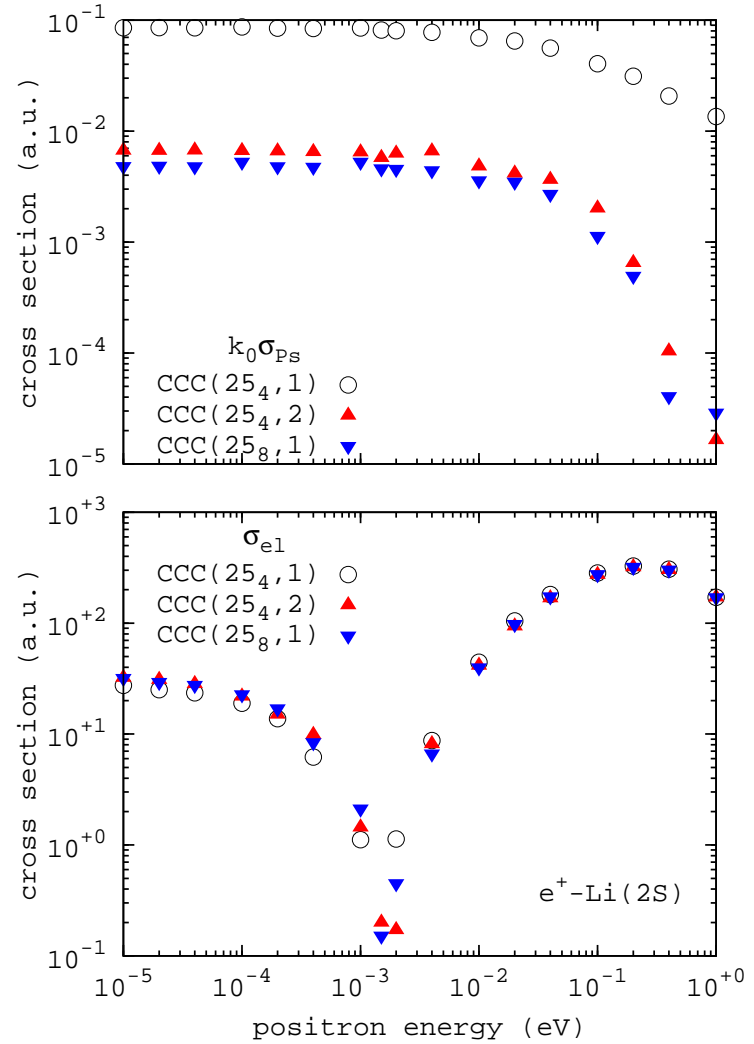

FIG. 1: (Color online) $\sigma_{\text {el }}$ (lower panel) and $k_{0} \sigma_{\mathrm{Ps}}$ (upper panel) for positron-lithium scattering, as a function of the incident positron energy $\left(13.6 k_{0}^{2}\right)$, for the zeroth partial wave calculated with the indicated $\operatorname{CCC}\left(N_{l_{\max }^{(\mathrm{Li})}}, N_{l_{\max }}^{(\mathrm{Ps})}\right)$ Laguerre basis parameters, see text.

extraordinary, as it says that the inclusion of the closed lithium states with $5 \leq l \leq 8$ has such a dramatic reduction of the $\mathrm{Ps}(1 \mathrm{~s})$ cross sections, though almost no effect on the elastic ones. The presented $\operatorname{CCC}\left(25_{4}, 2\right)$ calculations show that most of the very large effect of the large- $l$ lithium states can be taken into account by the addition of just the single closed $\operatorname{Ps}(2 \mathrm{~s})$ state. In other words, the large- $l$ atomic states are essentially taking into account the effect of virtual $\operatorname{Ps}(2 s)$ formation on the $\operatorname{Ps}_{\mathrm{s}}(1 \mathrm{~s})$ cross section. For completeness, though not presented, the addition of $\operatorname{Ps}(3 \mathrm{~s})$ yields barely distinguishable results from $\operatorname{CCC}\left(25_{8}, 1\right)$, confirming the required internal consistency.

Fig. 2 shows $k_{0} \sigma_{\mathrm{Ps}}$ (upper panel) and $\sigma_{\mathrm{el}}$ (lower panel) for positron-sodium scattering. Once again the $\operatorname{CCC}(258,1)$ calculations represent the benchmark results for the zeroth partial wave, and again a clear linear dependence of $\sigma_{\mathrm{Ps}}$ on $k_{0}$ at the very low energies is established. Unlike the case of lithium, the $\operatorname{CCC}\left(25_{4}, 1\right)$ results (not shown) are already not far from the convergent ones. So instead, to demonstrate internal consistency, we start from the much smaller $\operatorname{CCC}\left(25_{1}, 1\right)$ calculations, which yield results almost an order of magnitude too large for the $\operatorname{Ps}(1 \mathrm{~s})$ cross section, and substantially smaller for elastic scattering. Given that the atomic states with
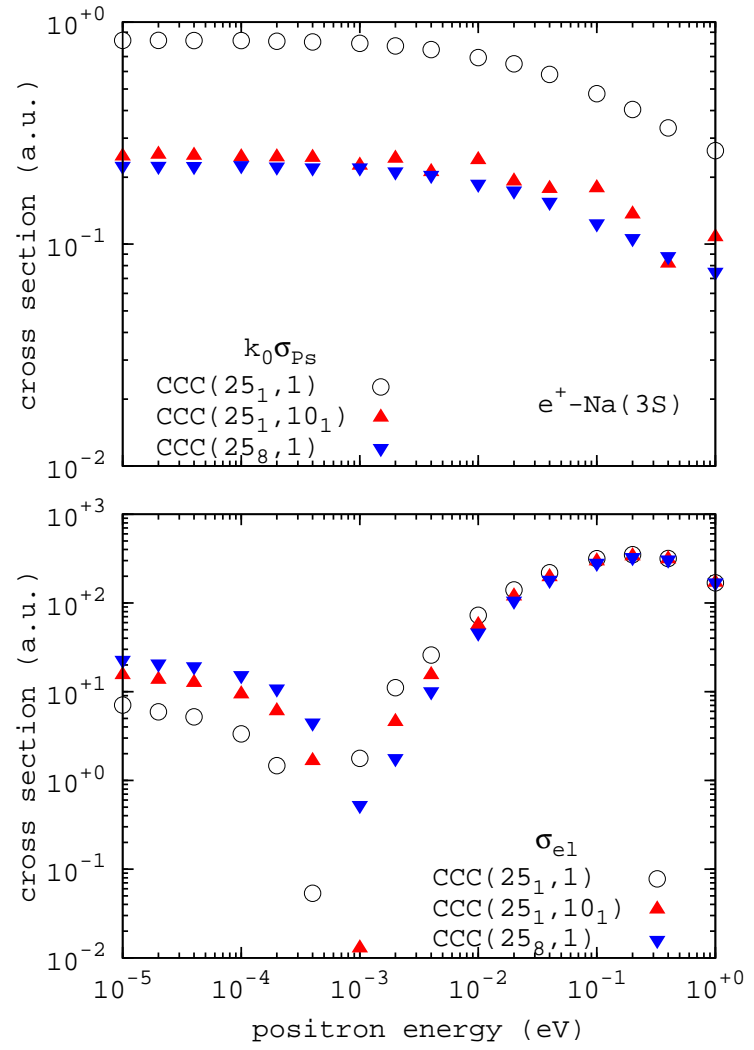

FIG. 2: (Color online) The same as Fig. 1 but for positronsodium scattering.

$2 \leq l \leq 8$ have been dropped it is not surprising to see such a difference. An interesting question is whether the convergent results can be recovered by just adding more Ps states. We found that indeed this can be achieved by setting $l_{\max }^{(\mathrm{Ps})}=1$, and increasing $N_{l}^{(\mathrm{Ps})}$ to convergence around $N_{l}^{(\mathrm{Ps})}=10-l$. These calculations are labeled $\operatorname{CCC}\left(25_{1}, 10_{1}\right)$, and include 10 Ps s-states and 9 Ps pstates of both negative and positive energies.

In Fig. 3 similar results for positron-potassium scattering are presented. Again, it is the $\operatorname{CCC}\left(25_{8}, 1\right)$ calculations that are convergent, and demonstrate the linear behavior of $\sigma_{\mathrm{Ps}}$ at the lowest energies. Here, as for lithium, we demonstrate internal consistency by starting with the $\operatorname{CCC}\left(25_{4}, 1\right)$ results. These are an order of magnitude above the convergent results for the $\operatorname{Ps}(1 \mathrm{~s})$ cross sections, though hardly different for the elastic cross sections. This time just adding the closed $\mathrm{Ps}_{\mathrm{s}}(2 \mathrm{~s})$ state (not shown) has little effect on the results. However, taking $l_{\max }^{(\mathrm{Ps})}=1$ and setting the Laguerre basis size to $N_{l}^{(\mathrm{Ps})}=5-l$ we find that the $\operatorname{CCC}\left(25_{4}, 5_{1}\right)$ calculations yield the converged results. So in this case considerably more Ps states were necessary to recover the convergent results than for lithium.

It is the complex interplay of atomic and core polarizability, which varies substantially for each alkali atom, that leads to such a variety of convergence behavior. It is also interesting to observe other variations. For ex- 

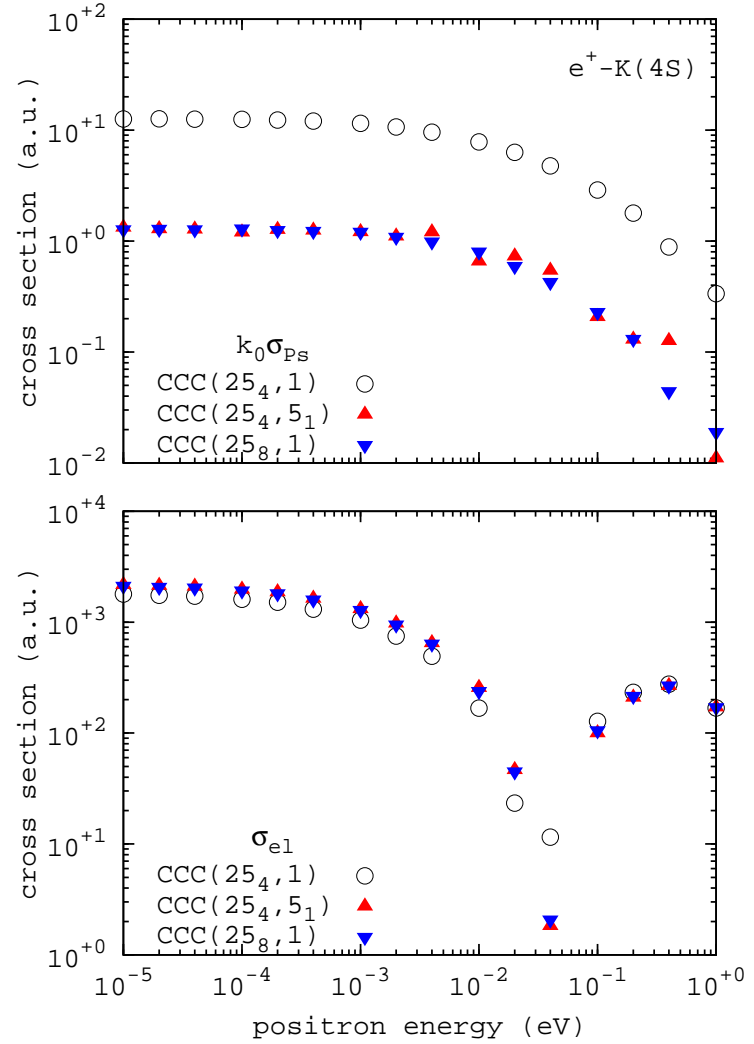

FIG. 3: (Color online) The same as Fig. 1 but for positronpotassium scattering.

ample, for both $\mathrm{Li}$ and $\mathrm{Na}$ there is a minimum in the elastic cross section around $0.001 \mathrm{eV}$, yet for $\mathrm{K}$ it moves to around $0.04 \mathrm{eV}$. The magnitude of the energy elastic cross section (determines the scattering length) rises rapidly from $\mathrm{Li}$ to $\mathrm{K}$, as does $k_{0} \sigma_{\mathrm{Ps}}$.

\section{CONCLUSION}

In conclusion, we have established convergent elastic and Ps-formation cross sections at very low energy positron scattering on the considered alkali targets. Utilising the interplay between states on either the atomic, or the Ps center, internal consistency has been demonstrated. Much larger calculations were required for convergence than previously reported $[5,25]$, with the smaller calculations yielding results in error by as much as an order of magnitude. The $1 / k_{0}$ threshold behavior for the Ps-formation cross section has been clearly observed. It is interesting to note that this threshold behavior is the same in the present smallest non-convergent calculations, as well as the much smaller ones performed earlier [5, 25]. Clearly, establishing the functional behavior is much easier than the absolute cross sections.

Lastly, we have demonstrated the internal consistency of the calculations. The fact that the atomic and Ps states are not orthogonal, and yet both form (near) complete expansions, ensures that the two-center closecoupling approach is fundamentally ill-conditioned. Consequently, this does not allow for an arbitrary increase in the number of expansion states used, not only in the problems considered, but more generally wherever multicenter collision problems appear. Hence considerable care needs to be undertaken to demonstrate convergence as has been done here.

\section{Acknowledgments}

We thank Michael Bromley for the suggestion of looking at the problem, and his useful comments on the manuscript. The support of the Australian Research Council, the Australian National Computational Infrastructure Facility and its Western Australian node iVEC, is gratefully acknowledged.

[1] E. P. Wigner, Phys. Rev. 73, 1002 (1948).

[2] H. R. Sadeghpour, J. L. Bohn, M. J. Cavagnero, B. D. Esry, I. I. Fabrikant, J. H. Macek, and A. R. P. Rau, Journal of Physics B: Atomic, Molecular and Optical Physics 33, R93 (2000).

[3] I. Dumitriu, R. C. Bilodeau, T. W. Gorczyca, C. W. Walter, N. D. Gibson, Z. D. Pesic, D. Rolles, and N. Berrah, Physical Review A 82, 043434 (2010).

[4] G. Laricchia, J. Moxom, M. Charlton, . Kövér, and W. E. Meyerhof, Hyperfine Interactions 89, 209 (1994).

[5] M. T. McAlinden, A. A. Kernoghan, and H. R. J. Walters, Hyperfine Interact. 89, 161 (1994).

[6] A. S. Kadyrov, I. Bray, and A. T. Stelbovics, Physical Review Letters 98, 263202 (2007).
[7] R. Barrachina, J. Fiol, and P. Macri, Nuclear Instruments and Methods in Physics Research Section B: Beam Interactions with Materials and Atoms 266, 402 (2008).

[8] B. Jonson, Physics Reports 389, 1 (2004).

[9] B. DeMarco, J. L. Bohn, J. P. Burke, M. Holland, and D. S. Jin, Physical Review Letters 82, 4208 (1999).

[10] Y. P. Zhang, C. H. Cheng, J. T. Kim, J. Stanojevic, and E. E. Eyler, Physical Review Letters 92, 203003 (2004).

[11] M. S. T. Watts and J. W. Humberston, Journal of Physics B: Atomic, Molecular and Optical Physics 25, L491 (1992).

[12] J. W. Humberston and M. S. T. Watts, Hyperfine Interactions 89, 47 (1994), ISSN 0304-3834, 1572-9540.

[13] E. A. G. Armour and C. W. Chamberlain, Journal of 
Physics B: Atomic, Molecular and Optical Physics 35, L489 (2002).

[14] J. Mitroy, Aust. J. Phys. 48, 893 (1995).

[15] R. N. Hewitt, C. J. Noble, and B. H. Bransden, Journal of Physics B: Atomic, Molecular and Optical Physics 26, 3661 (1993).

[16] M. T. McAlinden, A. A. Kernoghan, and H. R. J. Walters, Journal of Physics B: Atomic, Molecular and Optical Physics 29, 555 (1996).

[17] C. Campbell, M. T. McAlinden, A. A. Kernoghan, and H. Walters, Nuclear Instruments and Methods in Physics Research Section B: Beam Interactions with Materials and Atoms 143, 41 (1998).

[18] G. Ryzhikh and J. Mitroy, Journal of Physics B: Atomic, Molecular and Optical Physics 30, 5545 (1997).

[19] A.-T. Le, M. W. J. Bromley, and C. D. Lin, Phys. Rev. A 71, 032713 (2005).

[20] S. J. Ward and J. Shertzer, Phys. Rev. A 68, 032720 (2003).

[21] S. J. Ward, J. Shertzer, S. Y. Ovchinnikov, and J. H. Macek, Phys. Rev. A 75, 012713 (2007).

[22] C. K. Kwan, W. E. Kauppila, R. A. Lukaszew, S. P. Parikh, T. S. Stein, Y. J. Wan, and M. S. Dababneh,
Phys. Rev. A 44, 1620 (1991).

[23] S. Zhou, S. P. Parikh, W. E. Kauppila, C. K. Kwan, D. Lin, A. Surdutovich, and T. S. Stein, Phys. Rev. Lett. 73, 236 (1994).

[24] E. Surdutovich, J. M. Johnson, W. E. Kauppila, C. K. Kwan, and T. S. Stein, Phys. Rev. A 65, 032713 (2002).

[25] A. A. Kernoghan, M. T. McAlinden, and H. R. J. Walters, Journal of Physics B: Atomic, Molecular and Optical Physics 27, L625 (1994), ISSN 0953-4075, 1361-6455.

[26] S. J. Ward and J. Shertzer, New Journal of Physics 14, 025003 (2012).

[27] J. Mitroy and G. G. Ryzhikh, Journal of Physics B: Atomic, Molecular and Optical Physics 34, 2001 (2001).

[28] J. Mitroy, M. W. J. Bromley, and G. G. Ryzhikh, J. Phys. B 35, R81 (2002).

[29] A. S. Kadyrov and I. Bray, Phys. Rev. A 66, 012710 (2002).

[30] A. V. Lugovskoy, A. S. Kadyrov, I. Bray, and A. T. Stelbovics, Phys. Rev. A 82, 062708 (2010).

[31] I. Bray, Phys. Rev. A 49, 1066 (1994).

[32] A. V. Lugovskoy, A. S. Kadyrov, I. Bray, and A. T. Stelbovics, Phys. Rev. A 85, 034701 (2012). 